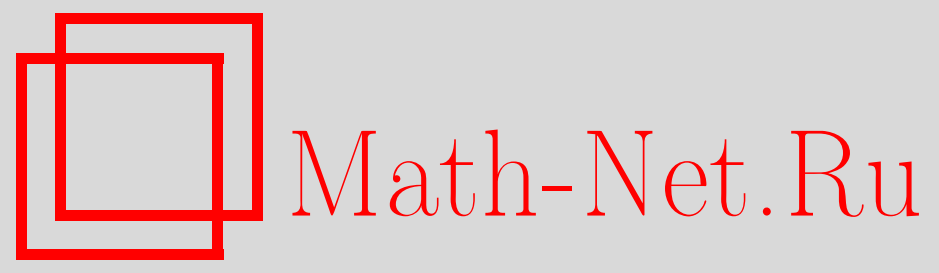

С. М. Буравлев, Повторения с точностью до перестановок, образующих латинский прямоугольник, Дискрет. матем., 2000, том 12, выпуск 1, 24-46

DOI: https://doi.org/10.4213/dm312

Использование Общероссийского математического портала Math-Net.Ru подразумевает, что вы прочитали и согласны с пользовательским соглашением http://www . mathnet.ru/rus/agreement

Параметры загрузки:

IP : 54.174 .149 .18

26 апреля 2023 г., 16:23:57 


\title{
Повторения с точностью до перестановок, образующих латинский прямоугольник
}

\author{
(C) 2000 г. C.M. Буравлев
}

\begin{abstract}
В работе изучаются предельные распределения случайных величин, связанных с повторениями с точностью до перестановок в последовательности независимых испытаний, причем множество перестановок образует латинский прямоугольник. Исследования проводятся в случае последовательности независимых испытаний в схеме серий. Ранее А.М. Зубковым и В.Г. Михайловым изучался случай точных повторений, автором - случай, когда соответствующее перестановкам множество подстановок совпадало с симметрической группой.
\end{abstract}

\section{1. Постановка задачи. Основные результаты}

Пусть $X=x_{1}, x_{2}, \ldots, x_{N}$ - последовательность независимых одинаково распределенных в каждой серии случайных величин, принимающих значения из множества $A=\{1,2, \ldots, v\}$ и

$$
\mathbf{P}\left(x_{i}=s\right)=p_{s}(N), \quad i=1, \ldots, N, \quad s=1, \ldots, v, \quad \bar{p}=\left(p_{1}(N), \ldots, p_{v}(N)\right)
$$

(в дальнейшем для краткости $p_{s}(N)$ обозначим через $p_{s}$ ), $\Omega$ - некоторое подмножество подстановок вида $\left(\begin{array}{ccc}1 & \ldots & v \\ \omega_{1} & \ldots & \omega_{v}\end{array}\right)$ симметрической группы $S_{v}, \Omega \subseteq S_{v}$.

Определим по $X_{i}(n)=\left(x_{i}, \ldots, x_{i+n-1}\right)$ и $X_{j}(n)=\left(x_{j}, \ldots, x_{j+n-1}\right)$ при $i \neq j$, $i, j=1, \ldots, N-n+1$, множество $Z\left(X_{i}(n), X_{j}(n)\right) \subseteq \Omega$ всех таких подстановок, что $x_{j+t}=Z\left(x_{i+t}\right)$ при $t=0, \ldots, n-1$ и любой $Z \in Z\left(X_{i}(n), X_{j}(n)\right)$.

Определим также случайные величины-индикаторы

$$
\eta_{i j}(\Omega, n)=1 \Longleftrightarrow Z\left(X_{i}(n), X_{j}(n)\right) \neq \varnothing .
$$

В работе А.М. Зубкова и В.Г. Михайлова [1] исследовались предельные распределения случайных величин, связанных с повторениями в последовательности независимых испытаний. В этом случае $\eta_{i j}(\Omega, n)=1$, если $x_{j+t}=x_{i+t}, t=0, \ldots, n-1$, т.е. связь знаков отрезков определялась одной тождественной подстановкой $E=$ $\left(\begin{array}{lll}1 & \ldots & v \\ 1 & \ldots & v\end{array}\right)$. В работе автора [2] исследовался случай $\Omega=S_{v}$.

В данной работе рассматривается ситуация, когда нижние строки подстановок $\Omega=\left\{Z_{1}, \ldots, Z_{k}\right\}$ образуют латинский прямоугольник, $\Omega=L_{k v}=\left\{Z_{1}, \ldots, Z_{k}\right\}$, $k \leqslant v$. (В латинском прямоугольнике в каждой строке и в каждом столбце все элементы различны (см. [3], с. 33)).

Подобный вид множества $\Omega$ возникает, например, при анализе траекторий случайных блужданий. Рассмотрим случайное блуждание $x_{1}, \ldots, x_{N}$ по множеству 
$\{0,1, \ldots, v-1\}$ наименьших неотрицательных вычетов по модулю $v$, т. е. случайный процесс, удовлетворяющий рекуррентным соотношениям

$$
x_{k+1}=x_{k}+\varepsilon_{k} \quad(\bmod v), \quad k=1, \ldots, N-1,
$$

где $\varepsilon_{1}, \ldots, \varepsilon_{N-1}$ - последовательность независимых одинаково распределенных случайных величин со значениями в $\{0,1, \ldots, v-1\}$.

Тогда, если $\varepsilon_{j+t}=\varepsilon_{i+t}, t=1, \ldots, n-1$, то

$$
x_{j+t}=Z\left(X_{i+t}\right), \quad t=1, \ldots, n,
$$

где

$$
Z=\left(\begin{array}{rr}
a & \\
a+x_{j}-x_{i} & (\bmod v)
\end{array}\right), \quad a=0, \ldots, v-1 .
$$

В этом случае множество $\Omega$ определяется различными степенями подстановок сдвига и их нижние строки образуют латинский квадрат.

Если $\Omega=L_{k v}$, то условие $\eta_{i j}\left(L_{k v}, n\right)=1$ однозначно определяет подстановку $Z$ и можно ввести индикаторы

$$
\tilde{\eta}_{i j}(\Omega, n)=1 \Longleftrightarrow Z\left(X_{i}(n), X_{j}(n)\right) \neq \varnothing
$$

и либо $\min (i, j)=1$, либо $x_{j-1} \neq Z\left(x_{i-1}\right)$ для любой $Z \in Z\left(X_{i}(n), X_{j}(n)\right)$.

В работе исследуются предельные распределения случайных величин:

$$
\begin{aligned}
\xi\left(L_{k v}, n, N\right) & =\sum_{1 \leqslant i<j \leqslant N-n+1} \eta_{i j}\left(L_{k v}, n\right), \quad \tilde{\xi}\left(L_{k v}, n, N\right)=\sum_{1 \leqslant i<j \leqslant N-n+1} \tilde{\eta}_{i j}\left(L_{k v}, n\right), \\
\tau\left(L_{k v}, n\right) & =\min \left\{N: \xi\left(L_{k v}, n, N\right)>0\right\}, \quad \mu\left(L_{k v}, N\right)=\max \left\{n: \xi\left(L_{k v}, n, N\right)>0\right\} .
\end{aligned}
$$

Положим

$$
\begin{aligned}
\psi & =\psi\left(L_{k v}, n, \bar{p}\right)=\sum_{t=1}^{k}\left(\mathbf{P}\left(Z_{t}\right)\right)^{n}\left(1-\mathbf{P}\left(\mathbf{Z}_{t}\right)\right) \\
\psi_{1} & =\psi_{1}\left(L_{k v}, n, \bar{p}\right)=\sum_{t=1}^{k}\left(\mathbf{P}\left(Z_{t}\right)\right)^{n}, \quad p^{*}=\max _{1 \leqslant s \leqslant v} p_{s}
\end{aligned}
$$

где

$$
\mathbf{P}\left(Z_{t}\right)=\sum_{s=1}^{v} p_{s} p_{Z_{t}(s)}, \quad t=1, \ldots, k .
$$

Теорема 1. Пусть $N \rightarrow \infty$, а величинь $n, k, v, p_{1}, \ldots, p_{v}$ и множество подстановок $L_{k v}$ меняются так, что

$$
C_{N}^{2} \psi\left(L_{k v}, n, \bar{p}\right) \rightarrow \lambda>0 .
$$

Тогда

(a) если

$$
\left(k\left(p^{*}\right)^{n}\right)^{2} \leqslant \psi_{1}\left(L_{k v}, n, \bar{p}\right),
$$

$u k n^{q} N\left(p^{*}\right)^{n} \rightarrow 0$ для любого натурального $q<\infty$, или 
(b) если для некоторого $\omega, 1 \leqslant \omega \leqslant v$, такого, что $p_{\omega}=p^{*}$,

$$
\left(p^{*}\right)^{n} \leqslant \sum_{t=1}^{k}\left(p_{Z_{t}(\omega)}\right)^{n}
$$

и выполняется одна из двух совокупностей условий:

(1) $p^{*} \leqslant c<1 u(\max (k, n))^{q} N\left(p^{*}\right)^{n} \rightarrow 0$ для любого натуралъного $q<\infty$, или

(2) $p^{*} \rightarrow 1$ так, что для любого натурального $q<\infty$

$$
n^{q} k\left(p^{*}\right)^{n} N \rightarrow 0 \text { npu } \frac{k}{n}\left(1-p^{*}\right)^{-1 / 2}<1
$$

$u$

$$
n k^{2 q-1}\left(p^{*}\right)^{n}\left(1-p^{*}\right)^{1-q} N \rightarrow 0, n p u \frac{k}{n}\left(1-p^{*}\right)^{-1 / 2} \geqslant 1,
$$

то для любого фиксированного $s=0,1, \ldots$

$$
\mathbf{P}\left(\tilde{\xi}\left(L_{k v}, n, N\right)=s\right) \rightarrow \frac{\lambda^{s}}{s !} e^{-\lambda} .
$$

Условия (1) и (2) дают необходимые для доказательства теоремы соотношения между вероятностными свойствами латинского прямоугольника и параметрами $n$, $v, k$ схемы. Заметим, что если $k=1$, то условие (1) принимает вид

$$
\left(p^{*}\right)^{2} \leqslant \mathbf{P}(Z)
$$

и оно выполняется, если $Z$ равна тождественной подстановке. Если нижние строки множества $L_{k v}$ образуют латинский квадрат, то условие (2) выполняется всегда. При $k=1$ оно выполняется, если для некоторого $\omega, 1 \leqslant \omega \leqslant v$, такого, что $p_{\omega}=p^{*}$, верно равенство $\omega=Z(\omega)$.

Теорема 2. Пусть $N \rightarrow \infty$, а величинъ $n, k, v, p_{1}, \ldots, p_{v}$ и множество подстановок $L_{k v}$ меняются так, что

$$
\begin{gathered}
p^{*} \leqslant c<1, \\
n^{q} N\left(p^{*}\right)^{n} \rightarrow 0 \text { для любого натуралъного } q<\infty, \\
\mathbf{P}\left(Z_{t}\right) \rightarrow \delta_{t} \in[0,1), \quad t=1, \ldots, k,
\end{gathered}
$$

подстановки в множестве $L_{k v}$ упорядоченъ так, что

$$
\delta=\delta_{1}=\ldots=\delta_{h}>\delta_{h+1} \geqslant \ldots \geqslant \delta_{k}, \quad 1 \leqslant h \leqslant k,
$$

$u$

$$
\begin{aligned}
C_{N}^{2}\left(\mathbf{P}\left(Z_{t}\right)\right)^{n}\left(1-\mathbf{P}\left(Z_{t}\right)\right) & \rightarrow \lambda_{t}>0, & & t=1, \ldots, h, \\
\left(p^{*}\right)^{2} & \leqslant \mathbf{P}\left(Z_{t}\right), & & t=1, \ldots, h .
\end{aligned}
$$

Тогда для $y<\delta^{-1} u k=1,2, \ldots$

$$
\mathbf{E} y^{\xi\left(L_{k v}, n, N\right)}=\prod_{t=1}^{h} \exp \left\{\frac{\lambda_{t}(y-1)}{1-\delta y}\right\}(1+o(1)) .
$$


Заметим, что условие $\left(p^{*}\right)^{2}<\mathbf{P}\left(Z_{t}\right), t=1, \ldots, h$, выполняется, например, если существуют попарно различные числа $\omega_{1}, \ldots, \omega_{h} \in\{1, \ldots, v\}$ такие, что $p_{\omega_{1}}=\ldots=$ $p_{\omega_{h}}=p^{*}$ и $Z_{t}\left(\omega_{t}\right)=\omega_{t}, t=1, \ldots, h$.

Из теоремы 2 следует, что вклад в $\xi\left(L_{k v}, n, N\right)$ вносят только строки, отвечающие подстановкам с максимальными вероятностями $\mathbf{P}(Z)$, соответствующие им случайные величины асимптотически независимы и имеют в пределе сложное распределение Пуассона.

Следствие 1. Пусть величинъ $n, k, v, p_{1}, \ldots, p_{v}$ и множество подстановок $L_{k v}$ меняются так, что

$$
\psi\left(L_{k v}, n, \bar{p}\right) \rightarrow 0
$$

$u$

$$
n^{q}\left(p^{*}\right)^{n} k\left(\psi\left(L_{k v}, n, \bar{p}\right)\right)^{-1 / 2} \rightarrow 0
$$

для любого натуралъного $q<\infty$, тогда

$$
\mathbf{P}\left(\tau\left(L_{k v}, n\right)\left(\psi\left(L_{k v}, n, \bar{p}\right)\right)^{-1 / 2} \leqslant x\right) \rightarrow 1-\exp \left\{-x^{2} / 2\right\}
$$

для любого действительного $x>0$.

Теорема 3. Пусть $N \rightarrow \infty$, а величинь $k, v, p_{1}, \ldots, p_{v}$ и множество подстановок $L_{k v}$ меняются так, что

$$
\begin{aligned}
\left(\frac{1}{2}-\frac{\ln p^{*}}{\ln \mathbf{P}\left(Z_{m}\right)}\right) \frac{\ln N}{\ln \ln N} & =\gamma(N) \rightarrow-\infty, \quad \frac{k}{\ln N} \rightarrow 0 \\
\psi\left(L_{k v}, 1, \bar{p}\right) & =\mathbf{P}\left(Z_{m}\right)\left(1-\mathbf{P}\left(Z_{m}\right)\right)(1+o(1))
\end{aligned}
$$

для некоторого $m, 1 \leqslant m \leqslant k$. Тогда

(a) ecлu $\mathbf{P}\left(Z_{m}\right) \rightarrow 1$, mo

$$
\mathbf{P}\left(\left(1-\mathbf{P}\left(Z_{m}\right)\right) \mu\left(L_{k v}, N\right)-\ln \left(C_{N}^{2}\left(1-\mathbf{P}\left(Z_{m}\right)\right)\right)<x\right) \rightarrow \exp \left\{-e^{-x}\right\},
$$

(b) если $\mathbf{P}\left(Z_{m}\right) \rightarrow \delta \in(0,1)$ и выполнены условия (1) или (2), то

$$
\mathbf{P}\left(\mu\left(L_{k v}, N\right)-\left[\frac{2 \ln N}{\left|\ln \mathbf{P}\left(Z_{m}\right)\right|}\right]<d\right)=\exp \left\{-\frac{1-\delta}{2} \delta^{2-\left\{2 \ln N /\left|\ln \mathbf{P}\left(Z_{m}\right)\right|\right\}}\right\}+o(1)
$$

(c) если $\mathbf{P}\left(Z_{m}\right) \rightarrow 0$ и выполнены условия (1) или (2), то

$$
\begin{aligned}
& \mathbf{P}\left(\mu\left(L_{k v}, N\right)=\left\langle\frac{2 \ln N}{\left|\ln \mathbf{P}\left(Z_{m}\right)\right|}\right\rangle+1\right) \rightarrow e^{-\lambda}, \\
& \mathbf{P}\left(\mu\left(L_{k v}, N\right)=\left\langle\frac{2 \ln N}{\left|\ln \mathbf{P}\left(Z_{m}\right)\right|}\right\rangle\right) \rightarrow 1-e^{-\lambda}
\end{aligned}
$$

npu

$$
\left(\frac{2 \ln N}{\left|\ln \mathbf{P}\left(Z_{m}\right)\right|}-\left\langle\frac{2 \ln N}{\left|\ln \mathbf{P}\left(Z_{m}\right)\right|}\right\rangle\right)\left|\ln P\left(Z_{m}\right)\right| \rightarrow \ln (2 \lambda), \quad 0<\lambda<\infty,
$$

$u$

$$
\mathbf{P}\left(\mu\left(L_{k v}, N\right)=\left[\frac{2 \ln N}{\left|\ln P\left(Z_{m}\right)\right|}\right]\right) \rightarrow 1
$$


$n p u$

$$
\left|\left(\frac{2 \ln N}{\left|\ln \mathbf{P}\left(Z_{m}\right)\right|}-\left\langle\frac{2 \ln N}{\left|\ln \mathbf{P}\left(Z_{m}\right)\right|}\right\rangle\right)\right|\left|\ln P\left(Z_{m}\right)\right| \rightarrow \infty
$$

$u$

$$
\exp \left\{\left\{\frac{2 \ln N}{\left|\ln \mathbf{P}\left(Z_{m}\right)\right|}\right\}\left|\ln P\left(Z_{m}\right)\right|\right\}=o\left(\ln ^{b} N\right), \quad 0<b<\infty .
$$

Положим

$$
\lambda(N)=C_{N}^{2} \psi\left(L_{k v}, n, \bar{p}\right)
$$

Теорема 4. Пусть $N \rightarrow \infty$, а величины $n, k, v, p_{1}, \ldots, p_{V}$ и множество подстановок $L_{k v}$ меняются так, что

$$
\begin{gathered}
\lambda(N) \rightarrow \infty, \quad \lambda(N) \ln \lambda(N)=o(\ln N), \\
k\left(p^{*}\right)^{n}=O\left(\frac{1}{N^{1+\delta}}\right) \text { для некоторого } 0<\delta<1 .
\end{gathered}
$$

Тогда

(а) если выполнено условие (2) и

$$
\begin{aligned}
& \lambda(N) n^{-2} \rightarrow \infty \\
& \lambda(N) k^{-1} \rightarrow \infty
\end{aligned}
$$

или

(b) если выполнено условие (1) и

$$
\lambda(N) n^{-1} \rightarrow \infty
$$

то равномерно по $s=1, \ldots, 2[\lambda(N)]$

$$
\mathbf{P}\left(\tilde{\xi}\left(L_{k v}, n, N\right)=s\right)=\frac{\lambda^{s}(N) e^{-\lambda(N)}}{s !}(1+o(1)) .
$$

Следствие 2. В условиях теоремы 4

$$
\lim _{N \rightarrow \infty} \mathbf{P}\left(\frac{\tilde{\xi}\left(L_{k v}, n, N\right)-\lambda(N)}{\sqrt{\lambda(N)}}<x\right)=\Phi(x) .
$$

Рассмотрим случайные величины

$$
\begin{aligned}
& \xi^{(\neq)}\left(L_{k v}, n, N\right)=\sum_{1 \leqslant i \neq j \leqslant N-n+1} \eta_{i j}\left(L_{k v}, n\right), \\
& \tilde{\xi}^{(\neq)}\left(L_{k v}, n, N\right)=\sum_{1 \leqslant i \neq j \leqslant N-n+1} \tilde{\eta}_{i j}\left(L_{k v}, n\right) .
\end{aligned}
$$

Представим множество $L_{k v}$ в виде

$$
L_{k v}=L_{k^{\prime} v} \cup L_{k^{\prime \prime} v}, \quad k^{\prime}+k^{\prime \prime}=k,
$$


где $L_{k^{\prime} v}=\left\{Z_{1}^{\prime}, \ldots, Z_{k^{\prime}}^{\prime}\right\}-$ множество всех подстановок $Z \in L_{k v}$, для которых $Z^{-1} \in$ $L_{k v}, L_{k^{\prime \prime} v}=L_{k v} \backslash L_{k^{\prime} v}=\left\{Z_{1}^{\prime \prime}, \ldots, Z_{k^{\prime \prime}}^{\prime \prime}\right\}$.

Нетрудно заметить, что случайная величина $\xi^{(\neq)}\left(L_{k^{\prime} v}, n, N\right)$ имеет такое же распределение, как и случайная величина $2 \xi\left(L_{k^{\prime} v}, n, N\right)$. Поэтому из теоремы 2 следует, что случайная величина $\xi^{(\neq)}\left(L_{k v}, n, N\right)$ имеет в пределе такое же распределение, как

$$
2 \sum_{t=1}^{k^{\prime}} \xi\left(Z_{t}^{\prime}, n, N\right)+\sum_{t=1}^{k^{\prime \prime}} \xi\left(Z_{t}^{\prime \prime}, n, N\right)
$$

Аналогичные рассуждения справедливы для $\tilde{\xi}^{(\neq)}\left(L_{k v}, n, N\right)$.

\section{2. Доказательство теоремы 1}

При доказательстве будем использовать схему и оценки, изложенные в работах [1] и [2]. Напомним, что для доказательства сходимости распределения случайной величины $\tilde{\xi}\left(L_{k v}, n, N\right)$ к пуассоновскому достаточно показать (см. [4]), что выполнены следующие соотношения (для краткости случайные величины $\tilde{\eta}_{i j}\left(L_{k v}, n\right)$ будем обозначать через $\left.\tilde{\eta}_{i j}(n)\right)$ :

$$
\max _{i, j} \mathbf{P}\left(\tilde{\eta}_{i j}(n)=1\right) \rightarrow 0, \quad \sum_{1 \leqslant i<j \leqslant N-n+1} \mathbf{P}\left(\tilde{\eta}_{i j}(n)=1\right) \rightarrow \lambda
$$

для любого фиксированного целого $r \geqslant 2$

$$
\begin{gathered}
\sum_{I_{r}(n, N)} \mathbf{P}\left(\tilde{\eta}_{i_{1} j_{1}}(n)=\ldots=\tilde{\eta}_{i_{r} j_{r}}(n)=1\right) \rightarrow 0, \\
\sum_{I_{r}(n, N)} \mathbf{P}\left(\tilde{\eta}_{i_{1} j_{1}}(n)=1\right) \ldots \mathbf{P}\left(\tilde{\eta}_{i_{r} j_{r}}(n)=1\right) \rightarrow 0,
\end{gathered}
$$

где

$$
\begin{aligned}
& I_{r}(n, N)=\left\{\left\{\left(i_{k}, j_{k}\right), 1 \leqslant i_{k}<j_{k} \leqslant N-n+1, k=1, \ldots, r\right\}:\right. \\
& \left(i_{k_{1}}, j_{k_{1}}\right) \neq\left(i_{k_{2}}, j_{k_{2}}\right), \text { если } k_{1} \neq k_{2} \text { и хотя бы два из } 2 r \text { отрезков } \\
& \left.\left[i_{k}, \ldots, i_{k}+n-1\right],\left[j_{k}, \ldots, j_{k}+n-1\right] \text { пересекаются }\right\}
\end{aligned}
$$

- множество наборов упорядоченных пар; для каждого $r=2,3, \ldots$.

$$
\frac{\mathbf{P}\left(\tilde{\eta}_{i_{1} j_{1}}(n)=\ldots=\tilde{\eta}_{i_{k} j_{k}}(n)=1\right)}{\mathbf{P}\left(\tilde{\eta}_{i_{1} j_{1}}(n)=1\right) \ldots \mathbf{P}\left(\tilde{\eta}_{i_{k} j_{k}}(n)=1\right)} \rightarrow 1
$$

равномерно по всем наборам попарно различных пар натуральных чисел

$$
\alpha_{r}=\left\{\left(i_{k}, j_{k}\right), 1 \leqslant i_{k}<j_{k} \leqslant N-n+1, k=1, \ldots, r\right\} \notin I_{r}(n, N) .
$$

В нашем случае, если $\alpha_{r} \notin I_{r}(n, N)$, т. е. если $2 r$ отрезков попарно не пересекаются, то в силу независимости элементов последовательности $X$

$$
\mathbf{P}\left(\tilde{\eta}_{i_{1} j_{1}}(n)=\ldots=\tilde{\eta}_{i_{k} j_{k}}(n)=1\right)=\mathbf{P}\left(\tilde{\eta}_{i_{1} j_{1}}(n)=1\right) \ldots \mathbf{P}\left(\tilde{\eta}_{i_{k} j_{k}}(n)=1\right)
$$


и соотношение (14) есть точное равенство.

Для вероятности $\mathbf{P}\left(\tilde{\eta}_{i j}(n)=1\right)$ справедливы следующие оценки.

Если $j-i>n$, то

$$
\mathbf{P}\left(\tilde{\eta}_{i j}(n)=1\right)=\psi<\left(p^{*}\right)^{n} k .
$$

Если $j-i \leqslant n$, то по аналогии с леммой 2 из [2] величины $x_{i}, \ldots, x_{j-1}$ являются свободными (на них не накладывается никаких ограничений), а остальные величины $x_{j}, \ldots, x_{j+n-1}$ однозначно определяются свободными и некоторой подстановкой $Z$, заменяя вероятности их появления на $p^{*}$, получаем, что

$$
\begin{aligned}
\mathbf{P}\left(\tilde{\eta}_{i j}(n)=1\right) & <\left(p^{*}\right)^{n} \sum_{Z \in L_{k v}}\left(\left(1-\sum_{x_{i-1} \in A} p_{x_{i-1}} p_{Z}\left(x_{i-1}\right)\right) \sum_{\left(x_{i}, \ldots, x_{j-1}\right) \in X^{(j-i)}} p_{x_{i}} \ldots p_{x_{j-1}}\right) \\
& <\left(p^{*}\right)^{n} k \sum_{\left(x_{i}, \ldots, x_{j-1}\right) \in X^{(j-i)}} p_{x_{i}} \ldots p_{x_{j-1}}=\left(p^{*}\right)^{n} k,
\end{aligned}
$$

где $X^{(j-i)}-$ множество упорядоченных $(j-i)$-выборок элементов множества $A$.

Рассмотрим случай (а) теоремы 1.

Из оценок (15) и условий теоремы следует выполнение условия (11): так как $\mathbf{P}\left(\tilde{\eta}_{i j}(n)=1\right)<\left(p^{*}\right)^{n} k$ и $\psi<\left(p^{*}\right)^{n} k$, справедлива оценка

$$
\left|\mathbf{P}\left(\tilde{\eta}_{i j}(n)=1\right)-\psi\right|<\left(p^{*}\right)^{n} k,
$$

поэтому

$$
\begin{aligned}
\sum_{1 \leqslant i<j \leqslant N-n+1} \mathbf{P}\left(\eta_{i j}(n)=1\right) & =C_{N}^{2} \psi+\sum_{1 \leqslant i<j \leqslant N-n+1, j-i \leqslant n}\left(\mathbf{P}\left(\tilde{\eta}_{i j}(n)=1\right)-\psi\right) \\
& =C_{N}^{2} \psi+O\left(k n N\left(p^{*}\right)^{n}\right) \rightarrow \lambda .
\end{aligned}
$$

Тем самым установлено, что

$$
\mathbf{E} \tilde{\xi}\left(L_{k v}, n, N\right)=C_{N}^{2} \psi\left(L_{k v}, n, \bar{p}\right)+O\left(k n N\left(p^{*}\right)^{n}\right)=\lambda+o(1) .
$$

Пусть

$$
I_{r}(n, N)=\bigcup_{a=0}^{r} I_{r}(a ; n, N),
$$

где $I_{r}(a ; n, N)=\left\{\alpha_{r} \in I_{r}(n, N)\right.$ : имеется ровно $a$ таких значений $s, 1 \leqslant s \leqslant r$, что $\left.i_{s}<j_{s} \leqslant j_{s}+n\right\} ;$ можно показать, что

$$
\left|I_{r}(a ; n, N)\right|=O\left(n^{a} N^{2 r-a}\right), \quad\left|I_{r}(0 ; n, N)\right|=O\left(n N^{2 r-1}\right)
$$

(cм. [1]).

Так как $\psi<\left(p^{*}\right)^{n} k$, то $2 \lambda<k N^{2}\left(p^{*}\right)^{n}$ при $\lambda>0$ и достаточно больших $N$, поэтому

$$
n / N<(2 \lambda)^{-1} k n N\left(p^{*}\right)^{n}=o(1) .
$$

Для слагаемых в (13) имеет место оценка

$$
\mathbf{P}\left(\tilde{\eta}_{i_{1} j_{1}}(n)=1\right) \ldots \mathbf{P}\left(\tilde{\eta}_{i_{r} j_{r}}(n)=1\right) \leqslant\left(\left(p^{*}\right)^{n} k\right)^{a} \psi^{r-a}
$$


поэтому с учетом оценок для мощности множеств $I_{r}(a ; n, N)$ и (17) находим, что

$$
\begin{aligned}
\sum_{I_{r}(n, N)} \mathbf{P}\left(\tilde{\eta}_{i_{1} j_{1}}=1\right) \ldots \mathbf{P}\left(\tilde{\eta}_{i_{r} j_{r}}=1\right) & \leqslant \sum_{a=0}^{r} \sum_{I_{r}(a ; n, N)}\left(\left(p^{*}\right)^{n} k\right)^{a} \psi^{r-a} \\
& =O\left(n N^{2 r-1} \psi^{r}\right)+\sum_{a=1}^{r} O\left(n^{a} N^{2 r-a}\left(p^{*}\right)^{a n} k^{a} \psi^{r-a}\right) \\
& =O\left(\frac{n}{N}\right)+\sum_{a=1}^{r} O\left(\left(k n N\left(p^{*}\right)^{n}\right)^{a}\right) \rightarrow 0 .
\end{aligned}
$$

Осталось проверить условие (12).

Сопоставим каждому набору $\alpha_{r}=\left\{\left(i_{s}, j_{s}\right), s=1, \ldots, r\right\}$ из $I_{r}(n, N)$ множество

$$
U\left(\alpha_{r}\right)=\bigcup_{s=1}^{r}\left(\left[i_{s}, i_{s}+n-1\right] \cup\left[j_{s}, j_{s}+n-1\right]\right),
$$

где $[l, t]=\{l, l+1, \ldots, t\}$. Обозначим через $A\left(\alpha_{r}\right)$ число связных компонент (отрезков) множества $U\left(\alpha_{r}\right)$. Очевидно, что $1 \leqslant A\left(\alpha_{r}\right) \leqslant 2 r-1$, если $\alpha_{r} \in I_{r}(n, N)$.

Определим множества

$$
I_{r}^{(a)}(n, N)=\left\{\alpha_{r} \in I_{r}(n, N): A\left(\alpha_{r}\right)=a\right\}, \quad 1 \leqslant a \leqslant 2 r-1,
$$

тогда (см. [1])

$$
I_{r}(n, N)=\bigcup_{a=1}^{2 r-1} I_{r}^{(a)}(n, N), \quad\left|I_{r}^{(a)}(n, N)\right|=O\left(n^{2 r-a} N^{a}\right) .
$$

Лемма 1. Если $\alpha_{r} \in I_{r}^{(a)}(n, N), r \geqslant 2,1 \leqslant a \leqslant 2 r-1, m o$

$$
\mathbf{P}\left(\alpha_{r}\right)=\mathbf{P}\left(\tilde{\eta}_{i_{1} j_{1}}(n)=\ldots=\tilde{\eta}_{i_{r} j_{r}}(n)=1\right)<\left(\psi_{1}\left(L_{k v}, n, \bar{p}\right)\right)^{(a-1) / 2}\left(p^{*}\right)^{n} k
$$

Доказательство. Доказательство проведем индукцией по числу $r$. Пусть $r=2$ и $\alpha_{2}=\left\{\left(i_{1}, j_{1}\right),\left(i_{2}, j_{2}\right)\right\} \in I_{r}^{(a)}(n, N), 1 \leqslant a \leqslant 3$.

Если $a=1$, то

$$
\mathbf{P}\left(\alpha_{2}\right)<\mathbf{P}\left(\eta_{i_{1} j_{1}}(n)=\eta_{i_{2} j_{2}}(n)=1\right)<\mathbf{P}\left(\eta_{i_{1} j_{1}}(n)=1\right),
$$

поэтому из оценок (15) следует, что $\mathbf{P}\left(\alpha_{2}\right)<\left(p^{*}\right)^{n} k$.

Необходимым условием для выполнения события $\left\{\tilde{\eta}_{i_{1} j_{1}}(n)=\tilde{\eta}_{i_{2} j_{2}}(n)=1\right\}$ является выполнение события $\left\{\eta_{i_{1} j_{1}}(n)=\eta_{i_{2} j_{2}}(n)=1\right\}$, которое, в свою очередь, равносильно выполнению равенств

$$
x_{i_{s}+t}=Z_{s}\left(x_{j_{s}+t}\right), \quad Z_{s} \in L_{k v}, \quad s=1,2, \quad t=0, \ldots, n-1 .
$$

Пусть $w$ - число различных неизвестных в системе (18). Пусть число свободных неизвестных в (18) равно $w^{\prime}$, обозначим их через $x_{t(1)}, \ldots, x_{t\left(w^{\prime}\right)}$. Тогда остальные однозначно определяются ими и некоторой парой подстановок $Z_{1}$ и $Z_{2}$, и таких пар существует не более $k^{2}$. Аналогично лемме 3 из [2] показывается, что при $a=2$ и $a=3$ в случае (b) (в обозначениях леммы 3 из [2]) число не свободных неизвестных 
равно $w-w^{\prime}=2 n$. Заменяя вероятности их появления на $p^{*}$, учитывая независимость элементов последовательности $X$ и неравенство (1), получаем, что

$$
\begin{aligned}
\mathbf{P}\left(\alpha_{2}\right) & <\mathbf{P}\left(\eta_{i_{1} j_{1}}(n)=\eta_{i_{2} j_{2}}(n)=1\right) \\
& <\left(p^{*}\right)^{2 n} \sum_{Z_{1} \in L_{k v}} \sum_{Z_{2} \in L_{k v}} \sum_{\left(x_{t(1)}, \ldots, x_{t\left(w^{\prime}\right)}\right) \in X^{\left(w^{\prime}\right)}} \mathbf{P}_{x_{t(1)}} \ldots \mathbf{P}_{x_{t\left(w^{\prime}\right)}} \\
& =\left(p^{*}\right)^{2 n} k^{2} \leqslant \psi_{1}^{1 / 2}\left(p^{*}\right)^{n} k .
\end{aligned}
$$

Появление симметричного случая расположения индексов $\left(i_{2}-i_{1}=j_{2}-j_{1}\right)$ невозможно в силу определения случайной величины $\tilde{\eta}_{i j}(n)$.

При $a=3$ помимо рассмотренного выше случая (b) возможны еще два случая.

Если одной из трех связных компонент $U\left(\alpha_{2}\right)$ является $\left[i_{1}, j_{1}+n-1\right]$ или $\left[i_{2}, j_{2}+n-1\right]$, то в силу неравенства $\psi<\psi_{1}$ и оценок $(15)$

$$
\mathbf{P}\left(\alpha_{2}\right)=\mathbf{P}\left(\tilde{\eta}_{i_{1} j_{1}}(n)=1\right) \mathbf{P}\left(\tilde{\eta}_{i_{2} j_{2}}(n)=1\right)<\psi_{1}\left(p^{*}\right)^{n} k .
$$

Во втором случае, когда какие-нибудь два начала отрезков из пар $\left(i_{1}, j_{1}\right)$ и $\left(i_{2}, j_{2}\right)$ совпадают, например, $i_{1}=i_{2}, j_{1} \neq j_{2}$, справедливы неравенства

$$
\begin{aligned}
\mathbf{P}\left(\alpha_{2}\right) & <\mathbf{P}\left(\eta_{i_{1} j_{1}}(n)=\eta_{i_{2} j_{2}}(n)=1\right) \\
& <\sum_{Z_{1} \in L_{k v}} \sum_{Z_{2} \in L_{k v}} \sum_{\left(x_{i_{1}}, \ldots, x_{i_{1}+n-1}\right) \in X^{(n)}} \prod_{t=0}^{n-1} p_{x_{i_{1}+t}} p_{Z_{1_{1}\left(x_{i_{1}+t}\right)}} p_{Z_{2}\left(x_{i_{1}+t}\right)} \sum_{\sum_{Z_{1} \in L_{k v}}} \prod_{\left(x_{i_{1}}, \ldots, x_{i_{1}+n-1}\right) \in X^{(n)}}^{n-1} p_{x_{i_{1}+t}} p_{Z_{1}\left(x_{i_{1}+t}\right)}=\left(p^{*}\right)^{n} k \psi_{1} .
\end{aligned}
$$

Таким образом, при $r=2$ оценки для вероятности $\mathbf{P}\left(\alpha_{2}\right)$ получены. Предположим, что они выполняются для всех $\alpha_{r} \in I_{r}(n, N)$, и рассмотрим наборы индексов

$$
\alpha_{r+1}=\left\{\left(i_{s}, j_{s}\right), s=1, \ldots, r+1\right\} \in I_{r+1}(n, N) .
$$

Возможны следующие случаи:

(a) $a^{\prime}=A\left(\alpha_{r+1}\right) \leqslant A\left(\alpha_{r}\right)=a$;

(b) $a^{\prime}=a+2$;

(c) $a^{\prime}=a+1$.

В случаях (a) и (b) очевидно, что соответственно

$$
\begin{aligned}
& \mathbf{P}\left(\alpha_{r+1}\right) \leqslant \mathbf{P}\left(\alpha_{r}\right)<\psi_{1}^{(a-1) / 2}\left(p^{*}\right)^{n} k \leqslant \psi_{1}^{\left(a^{\prime}-1\right) / 2}\left(p^{*}\right)^{n} k \\
& \mathbf{P}\left(\alpha_{r+1}\right)=\mathbf{P}\left(\alpha_{r}\right) \mathbf{P}\left(\tilde{\eta}_{i_{r+1} j_{r+1}}(n)=1\right)<\psi_{1}^{(a-1) / 2} \psi_{1}\left(p^{*}\right)^{n} k=\psi_{1}^{\left(a^{\prime}-1\right) / 2}\left(p^{*}\right)^{n} k .
\end{aligned}
$$

Рассмотрим случай (c). Если

$$
\left[i_{r+1}, i_{r+1}+n-1\right] \cap\left[j_{r+1}, j_{r+1}+n-1\right] \neq \varnothing,
$$


то с учетом (1) находим, что

$$
\mathbf{P}\left(\alpha_{r+1}\right)=\mathbf{P}\left(\alpha_{r}\right) \mathbf{P}\left(\tilde{\eta}_{i_{r+1} j_{r+1}}(n)=1\right)<\mathbf{P}\left(\alpha_{r}\right)\left(p^{*}\right)^{n} k \leqslant \mathbf{P}\left(\alpha_{r}\right) \psi_{1}^{1 / 2}<\psi_{1}^{\left(a^{\prime}-1\right) / 2}\left(p^{*}\right)^{n} k .
$$

\section{Пусть}

$$
\left[i_{r+1}, i_{r+1}+n-1\right] \cap\left[j_{r+1}, j_{r+1}+n-1\right]=\varnothing, \quad s=\left|U\left(\alpha_{r}\right) \cap\left[i_{r+1}, i_{r+1}+n-1\right]\right| .
$$

Предположим также, что пересечение $U\left(\alpha_{r}\right)$ с отрезком $\left[i_{r+1}, i_{r+1}+n-1\right]$ будет по точкам $i_{r+1}, \ldots, i_{r+1}+s-1,0<s \leqslant n\left(i_{r}=i_{r+1}\right.$ при $\left.s=n\right)$.

Величины $x_{j_{r+1}}, \ldots, x_{j_{r+1}+s-1}$ определяются величинами $x_{i_{r+1}}, \ldots, x_{i_{r+1}+s-1}$ и некоторой подстановкой, величины $x_{i_{r+1}+s}, \ldots, x_{i_{r+1}+n-1}$ являются свободными. Заменяя вероятности появления величин $x_{i_{r+1}}, \ldots, x_{i_{r+1}+s-1}$ на $p^{*}$, получаем, что

$$
\begin{aligned}
\mathbf{P}\left(\alpha_{r+1}\right) & =\mathbf{P}\left(\alpha_{r}, \tilde{\eta}_{i_{r+1} j_{r+1}}(n)=1\right) \\
& \leqslant \mathbf{P}\left(\alpha_{r}\right)\left(p^{*}\right)^{s} \sum_{Z \in L_{k v}} \sum_{\left(x_{i_{r+1}+s}, \ldots, x_{i_{r+1}+n-1}\right) \in X^{(n)}} \prod_{t=s}^{n-1} p_{x_{i_{r+1}+t}} p_{Z\left(x_{i_{r+1}+t}\right)} \\
& <\mathbf{P}\left(\alpha_{r}\right)\left(p^{*}\right)^{s} \sum_{Z \in L_{k v}}(P(Z))^{n-s}<\mathbf{P}\left(\alpha_{r}\right)\left(p^{*}\right)^{n} k \leqslant \mathbf{P}\left(\alpha_{r}\right) \psi_{1}^{1 / 2}<\psi_{1}^{\left(a^{\prime}-1\right) / 2}\left(p^{*}\right)^{n} k .
\end{aligned}
$$

Случаи пересечения множества $U\left(\alpha_{r}\right)$ с $\left[j_{r+1}, j_{r+1}+n-1\right]$ и хвостом отрезка $\left[i_{r+1}, i_{r+1}+n-1\right] \mid$ рассматриваются аналогично.

Заметим, что в условиях теоремы $\psi^{-1 / 2}=O(N)$ и

$$
\frac{1}{1-\mathbf{P}\left(Z_{t}\right)}<\frac{1}{1-p^{*}}=\sum_{j=0}^{\infty}\left(p^{*}\right)^{j}=\sum_{j=0}^{n-1}\left(p^{*}\right)^{j}+\sum_{j=n}^{\infty}\left(p^{*}\right)^{j}<n+\frac{\left(p^{*}\right)^{n}}{1-p^{*}}
$$

Так как $\psi_{1}<\psi /\left(1-p^{*}\right)$ и $\left(p^{*}\right)^{n} \rightarrow 0$, из неравенства

$$
\frac{1}{1-p^{*}}\left(1-\left(p^{*}\right)^{n}\right)<n
$$

следует, что

$$
\frac{1}{1-p^{*}}=O(n), \quad \psi_{1}=O(n \psi) .
$$

Из этих оценок и леммы 1 вытекает, что

$$
\sum_{I_{r}(n, N)} \mathbf{P}\left(\alpha_{r}\right)=O\left(\sum_{a=1}^{2 r-1} n^{2 r-a} N^{a} \psi_{1}^{(a-1) / 2}\left(p^{*}\right)^{n} k\right)=O\left(n^{2 r-1} k N\left(p^{*}\right)^{n}\right) \rightarrow 0
$$

Случай (a) теоремы 1 рассмотрен.

Рассмотрим случай (b).

Поскольку для вероятности $\mathbf{P}\left(\tilde{\eta}_{i j}(n)=1\right)$ верны оценки (15) и в условиях теоремы $O\left(k n N\left(p^{*}\right)^{n}\right) \rightarrow 0$, проверка соотношений $(11),(13)$ и (14) проводится так же, как в случае (a). 
Лемма 2. Если $\alpha_{r} \in I_{r}^{(a)}(n, N), r \geqslant 2,1 \leqslant a \leqslant 2 r-1$, mo

$$
\mathbf{P}\left(\alpha_{r}\right)<\left(\frac{\psi\left(L_{k v}, n, \bar{p}\right)}{1-p^{*}}\right)^{(a-1) / 2}\left(p^{*}\right)^{n} k^{a} .
$$

Доказательство. С учетом (2), справедливы оценки

$$
\begin{aligned}
\psi & =\sum_{t=1}^{k}\left(\left(\sum_{s=1}^{v} p_{s} p_{Z_{t}(s)}\right)^{n}\left(1-\sum_{s=1}^{v} p_{s} p_{Z_{t}(s)}\right)\right)>\left(1-p^{*}\right) \sum_{t=1}^{k}\left(\sum_{s=1}^{v} p_{s} p_{Z_{t}(s)}\right)^{n} \\
& >\left(1-p^{*}\right) \sum_{t=1}^{k}\left(p^{*} p_{Z_{t}(\omega)}\right)^{n}=\left(1-p^{*}\right)\left(p^{*}\right)^{n} \sum_{t=1}^{k}\left(p_{Z_{t}(\omega)}\right)^{n} \geqslant\left(1-p^{*}\right)\left(p^{*}\right)^{2 n}
\end{aligned}
$$

поэтому

$$
\left(p^{*}\right)^{n}<\left(\frac{\psi}{1-p^{*}}\right)^{1 / 2} .
$$

Далее доказательство леммы 2 проводится индукцией по $r$ аналогично доказательству леммы 1 с учетом соотношения (15) и последнего неравенства.

Из леммы 2 следует, что в условиях (b) теоремы

$$
\begin{aligned}
\sum_{I_{r}(n, N)} \mathbf{P}\left(\tilde{\eta}_{i_{1} j_{1}}(n)=\ldots\right. & \left.=\tilde{\eta}_{i_{r} j_{r}}(n)=1\right)=O\left(\sum_{a=1}^{2 r-1} n^{2 r-a} N^{a}\left(\frac{\psi}{1-p^{*}}\right)^{(a-1) / 2}\left(p^{*}\right)^{n} k^{a}\right) \\
& =O\left(n^{2 r}\left(\frac{\psi}{1-p^{*}}\right)^{-1 / 2}\left(p^{*}\right)^{n} \sum_{a=1}^{2 r-1}\left(\frac{k}{n\left(1-p^{*}\right)^{1 / 2}}\right)^{a}\right) \rightarrow 0 .
\end{aligned}
$$

Случай (b) рассмотрен, и теорема 1 доказана.

\section{3. Доказательство теоремы 2}

Рассмотрим сначала случай $k=1$, т. е. $\Omega=\{Z\}$. В этом случае проходит доказательство, изложенное в работе [1] для случая $\Omega=\{E\}$. Таким образом, при $k=1$ и $d=1,2, \ldots$

$$
\begin{aligned}
\mathbf{P}(\xi(Z, n, N)=d) & =\sum_{s=1}^{d} C_{d-1}^{s-1}(1-\delta)^{s} \delta^{d-s} \frac{\lambda^{s}}{s !} e^{-\lambda}(1+o(1)), \\
\mathbf{P}\{\xi(Z, n, N)=0) & =e^{-\lambda}(1+o(1)), \\
\mathbf{E} y^{\xi(Z, n, N)} & \rightarrow \exp \left\{\frac{\lambda(y-1)}{1-\delta y}\right\},
\end{aligned}
$$

где $\mathbf{P}(Z) \rightarrow \delta \in[0,1), C_{N}^{2}(P(Z))^{n}(1-\mathbf{P}(Z)) \rightarrow \lambda$.

Из этих выражений и теоремы 1 вытекает, что предельные распределения случайных величин $\tilde{\xi}(Z, n, N)$ и $\xi(Z, n, N)$ совпадают, если $\delta=0$.

Пусть $k>1$ и $\delta \neq 0$. Тогда для выполнения условия (3) теоремы 2 необходимо, чтобы $n \rightarrow \infty$. В этом случае

$$
C_{N}^{2}\left(\mathbf{P}\left(Z_{t}\right)\right)^{n}\left(1-\mathbf{P}\left(Z_{t}\right)\right)=o(\lambda), \quad t=h+1, \ldots, k
$$


и

$$
\mathbf{P}\left(\xi\left(L_{k v}, n, N\right)=d\right)=\mathbf{P}\left(\xi\left(L_{k v}, n, N\right)=d\right)(1+o(1)), \quad d=0,1,2, \ldots
$$

Докажем асимптотическую независимость случайных величин $\xi\left(Z_{1}, n, N\right), \ldots$, $\xi\left(Z_{h}(n, N)\right.$, когда подстановки $Z_{1}, \ldots, Z_{h}$ принадлежат одному латинскому прямоугольнику.

Рассмотрим совместное распределение случайных величин $\xi\left(Z_{1}, n, N\right), \ldots$, $\xi\left(Z_{h}(n, N)\right.$. Для упрощения записи введем обозначения

$$
\begin{gathered}
\left\{\gamma_{i_{c} j_{c}}\left(Z_{t}, n\right)=d_{c}^{(t)}, c=1, \ldots, r_{t}\right\}=\left\{\bar{\gamma}\left(Z_{t}, r_{t}, d^{(t)}\right\}\right. \\
\sum_{c=1}^{r_{t}} d_{c}^{(t)}=d^{(t)}, \quad t=1, \ldots, h, \quad \sum_{t=1}^{h} d^{(t)}=d .
\end{gathered}
$$

Тогда, для $d=1,2, \ldots$

$$
\begin{aligned}
& \mathbf{P}\left(\xi\left(Z_{t}, n, N\right)=d^{(t)}, t=1, \ldots, h\right) \\
& \quad=\sum_{r_{1}=1}^{d^{(1)}} \sum_{\substack{d_{1}^{(1)}+\ldots+d_{r_{1}}^{(1)}=d^{(1)} \\
d_{1}^{(1)}, \ldots, d_{r_{1}}^{(1)}>0}} \sum_{\alpha_{r_{1}}} \ldots \sum_{\substack{d_{h}=1 \\
d^{(h)}}} \sum_{\begin{array}{c}
(h)+\ldots+d_{r_{h}}^{(h)}=d^{(h)} \\
d_{1}^{(h)}, \ldots, d_{r_{h}}^{(h)}>0
\end{array}} \sum_{\alpha_{r_{h}}} \mathbf{P}\left(\bar{\gamma}\left(Z_{t}, r_{t}, d^{(t)}\right), t=1, \ldots, h\right) \\
& \quad=\sum_{r_{1}, \alpha_{r_{1}}} \ldots \sum_{r_{h}, \alpha_{r_{h}}} \mathbf{P}\left(\bar{\gamma}\left(Z_{t}, r_{t}, d^{(t)}\right), t=1, \ldots, h\right) .
\end{aligned}
$$

(Условное обозначение в последнем равенстве также применено для упрощения записи.)

В суммах (20) возможны следующие случаи взаимного расположения индексов в множествах $\alpha_{r_{t}}, t=1, \ldots, h$ :

(1) $\exists r^{\prime}, r^{\prime \prime} \in\left\{r_{1}, \ldots, r_{h}\right\}, r^{\prime} \neq r^{\prime \prime}: \alpha_{r^{\prime}} \cap \alpha_{r^{\prime \prime}} \neq \varnothing$

(2) $\forall r^{\prime}, r^{\prime \prime} \in\left\{r_{1}, \ldots, r_{h}\right\}, r^{\prime} \neq r^{\prime \prime}: \alpha_{r^{\prime}} \cap \alpha_{r^{\prime \prime}} \neq \varnothing$.

Первый случай означает, что существуют общие пары индексов, принадлежащие разным множествам пар индексов $\alpha_{r^{\prime}}$ и $\alpha_{r^{\prime \prime}}$. В этом случае вероятность, стоящая внутри сумм в (20), равна нулю, так как подстановки $Z_{1}, \ldots, Z_{h}$ принадлежат одному латинскому прямоугольнику и знаки двух отрезков не могут быть связаны разными подстановками.

Рассмотрим второй случай. Введем следующие обозначения:

$$
\begin{aligned}
& U_{d}\left(\alpha_{r}\right)= \bigcup_{s=1}^{h}\left(\left[i_{s}, i_{s}+n+d-1\right] \cup\left[j_{s}, j_{s}+n+d-1\right]\right), \\
& J(h, d)=\left\{\alpha_{r_{t}}, t=1, \ldots, h: \forall r^{\prime}, r^{\prime \prime} \in\left\{r_{1}, \ldots, r_{h}\right\}, r^{\prime} \neq r^{\prime \prime}: \alpha_{r^{\prime}} \cap \alpha_{r^{\prime \prime}}=\varnothing\right. \\
&\text { и } \left.U_{d}\left(\alpha_{r^{\prime}}\right) \cap U_{d}\left(\alpha_{r^{\prime \prime}}\right)=\varnothing\right\}, \\
& J^{*}(h, d)=\left\{\begin{array}{c}
\alpha_{r_{t}}, t=1, \ldots, h: \forall r^{\prime}, r^{\prime \prime} \in\left\{r_{1}, \ldots, r_{h}\right\}, r^{\prime} \neq r^{\prime \prime}, \alpha_{r^{\prime}} \cap \alpha_{r^{\prime \prime}}=\varnothing \\
\text { и } \left.\exists b^{\prime}, b^{\prime \prime} \in\left\{r_{1}, \ldots, r_{h}\right\}, b^{\prime} \neq b^{\prime \prime}: U_{d}\left(\alpha_{b^{\prime}}\right) \cap U_{d}\left(\alpha_{b^{\prime \prime}}\right)=\varnothing\right\} .
\end{array}\right.
\end{aligned}
$$


Учитывая, что при $\left\{\alpha_{r_{t}}, t=1, \ldots, h\right\}=J(h, d)$ выполняется равенство

$$
\mathbf{P}\left(\tilde{\gamma}\left(Z_{t}, r_{t}, d^{(t)}\right), t=1, \ldots, h\right)=\prod_{t=1}^{h} \mathbf{P}\left(\tilde{\gamma}\left(Z_{t}, r_{t}, d^{(t)}\right)\right.
$$

представим выражение (20) следующим образом:

$$
\begin{aligned}
& \sum_{r_{1}, \alpha_{r_{1}}} \ldots \sum_{r_{h}, \alpha_{r_{h}}} \mathbf{P}\left(\tilde{\gamma}\left(Z_{t}, r_{t}, d^{(t)}\right), t=1, \ldots, h\right) \\
& =\sum_{r_{1}} \ldots \sum_{r_{h}} \prod_{t=1}^{h} \mathbf{P}\left(\tilde{\gamma}\left(Z_{t}, r_{t}, d^{(t)}\right)\right)+\sum_{r_{1}} \ldots \sum_{r_{h}} \prod_{t=1}^{h} \mathbf{P}\left(\tilde{\gamma}\left(Z_{t}, r_{t}, d^{(t)}\right)\right. \\
& \quad+\sum_{r_{1}} \ldots \sum_{r_{h}} \mathbf{P}\left(\tilde{\gamma}\left(Z_{t}, r_{t}, d^{(t)}\right), t=1, \ldots, h\right)-\sum_{r_{1}} \ldots \sum_{r_{h}} \prod_{t=1}^{h} \mathbf{P}\left(\tilde{\gamma}\left(Z_{t}, r_{t}, d^{(t)}\right)\right) \\
& =\prod_{t=1}^{h} \mathbf{P}\left(\xi\left(Z_{t}, n, N\right)=d^{(t)}\right)+\sum_{r_{1}} \ldots \sum_{r_{h}} \mathbf{P}\left(\tilde{\gamma}\left(Z_{t}, r_{t}, d^{(t)}\right), t=1, \ldots, h\right) \\
& \quad-\sum_{r_{1}} \ldots \sum_{r_{h}} \prod_{t=1}^{h} \mathbf{P}\left(\tilde{\gamma}\left(Z_{t}, r_{t}, d^{(t)}\right)\right) .
\end{aligned}
$$

Покажем, что последние две суммы есть o(1). Рассмотрим первую из них. Верно следующее неравенство (смысл введенного условного обозначения аналогичен предыдущему):

$$
\begin{aligned}
& \mathbf{P}\left(\bar{\gamma}\left(Z_{t}, r_{t}, d^{(t)}\right), t=1, \ldots, h\right) \\
& \leqslant \mathbf{P}\left(\tilde{\eta}_{i_{1} j_{1}}\left(Z_{1}, n\right)=\ldots=\tilde{\eta}_{i_{r_{1}} j_{r_{1}}}\left(Z_{1}, n\right)=1, \ldots, \tilde{\eta}_{q_{1} s_{1}}\left(Z_{h}, n\right)=\ldots=\tilde{\eta}_{q_{r_{h}} s_{r_{h}}}\left(Z_{h}, n\right)=1\right) \\
& =\mathbf{P}\left(\bar{\eta}\left(Z_{t}, r_{t}\right), t=1, \ldots, h\right) .
\end{aligned}
$$

Поскольку в $J^{*}(h, d)$ множества $\alpha_{r_{t}}, t=1, \ldots, h$, попарно не пересекаются,

$$
J^{*}(h, d) \subseteq I_{r_{1}+\ldots+r_{h}}(n+d, N) .
$$

Поэтому, учитывая предыдущее неравенство, соотношения (22) и (19), получаем (для краткости полагаем $r_{1}+\ldots+r_{h}=r$ ), что

$$
\begin{aligned}
\sum_{r_{1}} \ldots & \sum_{r_{h}} \mathbf{P}\left(\bar{\gamma}\left(Z_{t}, r_{t}, d^{(t)}\right), t=1, \ldots, h\right) \\
=O & \left(\sum_{r_{1}=1}^{d^{(1)}} \ldots \sum_{r_{h}=1}^{d^{(h)}}\left(\begin{array}{c}
d^{(1)}-1 \\
r_{1}-1
\end{array}\right) \ldots\left(\begin{array}{c}
d^{(h)}-1 \\
r_{h}-1
\end{array}\right) \sum_{I_{r}(n+d, N)} \mathbf{P}\left(\bar{\gamma}\left(Z_{t}, r_{t}\right), t=1, \ldots, h\right)\right) \\
=O & \left(\sum_{r_{1}=1}^{d^{(1)}} \ldots \sum_{r_{h}=1}^{d^{(h)}}\left(\begin{array}{c}
d^{(1)}-1 \\
r_{1}-1
\end{array}\right) \ldots\left(\begin{array}{c}
d^{(h)}-1 \\
r_{h}-1
\end{array}\right)\right. \\
\quad & \sum_{I_{r}(n+d, N)} \mathbf{P}\left(\tilde{\eta}_{i_{1} j_{1}}\left(L_{h v}, n\right)=\ldots=\tilde{\eta}_{i_{r} j_{r}}\left(L_{h v}, n\right)=1\right)
\end{aligned}
$$




$$
\begin{aligned}
& =O\left(\sum_{r_{1}=1}^{d^{(1)}} \ldots \sum_{r_{h}=1}^{d^{(h)}}\left(\begin{array}{c}
d^{(1)}-1 \\
r_{1}-1
\end{array}\right) \ldots\left(\begin{array}{c}
d^{(h)}-1 \\
r_{h}-1
\end{array}\right)(n+d)^{2 r-1} N\left(p^{*}\right)^{n+d}\right) \\
& =O\left((n+d)^{2 d} N\left(p^{*}\right)^{n+d}\right)=o(1) .
\end{aligned}
$$

Рассмотрим последнюю сумму в (21). Так как

$$
\begin{aligned}
\mathbf{P}\left(\bar{\gamma}\left(Z_{t}, r_{t}, d^{(t)}\right)\right. & \leqslant \mathbf{P}\left(\tilde{\eta}_{i_{1} j_{1}}\left(Z_{1}, n\right)=\ldots=\tilde{\eta}_{i_{r_{t}} j_{r_{t}}}\left(Z_{t}, n\right)=1\right) \\
& =\mathbf{P}\left(\bar{\eta}\left(Z_{t}, r_{t}\right)\right), \quad t=1, \ldots, h,
\end{aligned}
$$

и

$$
J^{*}(h, d) \subseteq \bigcup_{t=1}^{h} \bigcup_{a_{t}=1}^{2 r_{t}} I_{r_{t}}^{\left(a_{t}\right)}(n+d, N), \quad a_{1}+\ldots+a_{h}<2 r
$$

то с учетом соотношений $\left|\dot{I}_{r}^{(a)}(n+d, N)\right|=O\left((n+d)^{2 r-a} N^{a}\right)$ для $1 \leqslant a \leqslant 2 r-1,(19)$ и (17) получаем, что

$$
\begin{aligned}
& \sum_{r_{1}} \ldots \sum_{r_{h}} \prod_{t=1}^{h} \mathbf{P}\left(\bar{\gamma}\left(Z_{t}, r_{t}, d^{(t)}\right)\right) \\
& =O\left(\sum_{r_{1}} \cdots \sum_{r_{h}} \prod_{t=1}^{h} \mathbf{P}\left(\bar{\gamma}\left(Z_{t}, r_{t}, d^{(t)}\right)\right)\right) \\
& =O\left(\sum_{r_{1}=1}^{d^{(1)}} \ldots \sum_{r_{h}=1}^{d^{(h)}}\left(\begin{array}{c}
d^{(1)}-1 \\
r_{1}-1
\end{array}\right) \ldots\left(\begin{array}{c}
d^{(h)}-1 \\
r_{h}-1
\end{array}\right) \sum_{\left\{\alpha_{r_{t}}, t=1, \ldots, h\right\}=J^{*}(h, d)} \prod_{t=1}^{h} \mathbf{P}\left(\bar{\eta}\left(Z_{t}, r_{t}\right)\right)\right) \\
& =O\left(\sum_{r_{1}=1}^{d^{(1)}} \ldots \sum_{r_{h}=1}^{d^{(h)}}\left(\begin{array}{c}
d^{(1)}-1 \\
r_{1}-1
\end{array}\right) \ldots\left(\begin{array}{c}
d^{(h)}-1 \\
r_{h}-1
\end{array}\right)\right. \\
& \left.\times \sum_{a_{1}=1}^{2 r_{1}} \ldots \sum_{a_{h}=1}^{2 r_{h}} \sum_{\alpha_{r_{1}} \in I_{r_{1}}^{\left(a_{1}\right)}(n+d, N)} \ldots \sum_{\alpha_{r_{h}} \in I_{r_{h}}^{\left(a_{h}\right)}(n+d, N)} \prod_{t=1}^{h} \mathbf{P}\left(\bar{\eta}\left(Z_{t}, r_{t}\right)\right)\right) \\
& =O\left(\sum_{r_{1}=1}^{d^{(1)}} \ldots \sum_{r_{h}=1}^{d^{(h)}}\left(\begin{array}{c}
d^{(1)}-1 \\
r_{1}-1
\end{array}\right) \ldots\left(\begin{array}{c}
d^{(h)}-1 \\
r_{h}-1
\end{array}\right)\right. \\
& \left.\times\left(\sum_{a_{1}=1}^{2 r_{1}} \ldots \sum_{a_{h}=1}^{2 r_{h}} \prod_{t=1}^{h}\left|I_{r_{t}}^{\left(a_{t}\right)}(n+d, N)\right| \mathbf{P}\left(\bar{\eta}\left(Z_{t}, r_{t}\right)\right)\right)\right) \\
& =O\left((n+d)^{2 d} N\left(p^{*}\right)^{n+d}\right)+O\left(\frac{n}{N}\right)=o(1) \text {. }
\end{aligned}
$$

Таким образом,

$$
\mathbf{P}\left(\xi\left(Z_{t}, n, N\right)=d^{(t)}, t=1, \ldots, h\right)=\prod_{t=1}^{h} \mathbf{P}\left(\xi\left(Z_{t}, n, N\right)=d^{(t)}\right)+o(1),
$$

т. е. случайные величины $\xi\left(Z_{t}, n, N\right), t=1, \ldots, h$, асимптотически независимы. 
Теорема 2 доказана.

Заметим, что аналогично показывается асимптотическая независимость случайных величин $\tilde{\xi}\left(Z_{t}, n, N\right), t=1, \ldots, k$.

\section{4. Доказательство следствия 1}

При любых $x \in(0, \infty)$

$$
\mathbf{P}\left(\tau\left(L_{k v}, n\right) \sqrt{\psi}>x\right)=\mathbf{P}\left(\xi\left(L_{k v}, n, N(x)\right)=0\right),
$$

где $N(x)=\left[x \psi^{-1 / 2}\right]$. Поскольку

$$
\mathbf{P}\left(\xi\left(L_{k v}, n, N\right)=0\right)=\mathbf{P}\left(\tilde{\xi}\left(L_{k v}, n, N\right)=0\right),
$$

достаточно доказать, что для случайной величины $\tilde{\xi}\left(L_{k v}, n, N(x)\right)$ справедлива теорема 1 с параметром $\lambda=x^{2} / 2$.

Так как $\psi \rightarrow 0$, то $N(x) \rightarrow \infty$,

$$
C_{N(x)}^{2} \psi=\frac{x^{2}}{2}(1+o(1))=\lambda(1+o(1))>0 .
$$

Далее из соотношения

$$
n^{q} N(x)\left(p^{*}\right)^{n} k=O\left(n^{q}\left(p^{*}\right)^{n} k \psi^{-1 / 2}\right) \rightarrow 0, \quad q<\infty,
$$

следует, что $\left(p^{*}\right)^{n} k<\psi^{1 / 2}<\psi_{1}^{1 / 2}$, т. е. неравенство (1) выполнено.

\section{5. Доказательство теоремы 3}

Рассмотрим случай (а). Если $\mathbf{P}\left(Z_{m}\right) \rightarrow 1$, то из неравенства $P\left(Z_{m}\right) \leqslant p^{*}$ следует, что $p^{*} \rightarrow 1, p_{Z_{m}(\omega)} \rightarrow 1$, где $p_{\omega}=p_{Z_{m}(\omega)}=p^{*}$ и условие $(2)$ выполнено. Таким образом, подстановка $Z_{m}$ переводит элемент $\omega$, имеющий максимальную вероятность $p^{*}$, в себя: $\omega=Z_{m}(\omega)$. Так как множество $L_{k v}$ есть латинский прямоугольник, подстановка $Z_{m}$ со свойством $\mathbf{P}\left(Z_{m}\right) \rightarrow 1$ единственна. Не ограничивая общности, положим $m=1$ и для краткости обозначим $Z_{1}=Z$.

Из (4) следует, что так как

$$
\psi\left(L_{k v}, 1, \bar{p}\right)=\mathbf{P}(Z)(1-\mathbf{P}(Z))\left(1+\sum_{t=2}^{k} \frac{\mathbf{P}\left(Z_{t}\right)\left(1-\mathbf{P}\left(Z_{t}\right)\right)}{\mathbf{P}(Z)(1-\mathbf{P}(Z))}\right),
$$

To

$$
\sum_{t=2}^{k} \frac{\mathbf{P}\left(Z_{t}\right)\left(1-\mathbf{P}\left(Z_{t}\right)\right)}{\mathbf{P}(Z)(1-\mathbf{P}(Z))}=o(1)
$$

и

$$
\begin{aligned}
\psi\left(L_{k v}, 1, \bar{p}\right) & =(\mathbf{P}(Z))^{n}(1-\mathbf{P}(Z))\left(1+\sum_{t=2}^{k}\left(\frac{\mathbf{P}\left(Z_{t}\right)}{\mathbf{P}(Z)}\right)^{n} \frac{1-\mathbf{P}\left(Z_{t}\right)}{1-\mathbf{P}(Z)}\right) \\
& =(\mathbf{P}(Z))^{n}(1-\mathbf{P}(Z))(1+o(1))
\end{aligned}
$$


для любого целого $n \geqslant 1$.

Положим

$$
n(x)=\left[\frac{x+\ln \left(C_{N}^{2}(1-\mathbf{P}(Z))\right)}{1-\mathbf{P}(Z)}\right]
$$

и проверим, что величины $n(x), k, v, p_{1}, \ldots, p_{v}$ удовлетворяют условиям теоремы 1 случаю (b2) с параметром $\lambda=e^{-x}$.

Так как $\mathbf{P}(Z) \rightarrow 1$, то $1-\mathbf{P}(Z) \sim-\ln \mathbf{P}(Z)$ и

$$
n(x) \sim-(\ln \mathbf{P}(Z))^{-1}\left(x+\ln \left(C_{N}^{2}(1-\mathbf{P}(Z))\right)\right),
$$

поэтому

$$
\begin{aligned}
C_{N}^{2} \psi\left(L_{k v}, n(x), \bar{p}\right) & \sim C_{N}^{2}(P(Z))^{n(x)}(1-\mathbf{P}(Z)) \\
& \sim C_{N}^{2}(1-\mathbf{P}(Z)) \exp \left\{-\left(x+\ln \left(C_{N}^{2}(1-\mathbf{P}(Z))\right)\right)\right\}=e^{-x}
\end{aligned}
$$

Далее, так как

$$
\mathbf{P}(Z)=\left(p^{*}\right)^{2}+\sum_{i=1}^{v} p_{i} p_{Z(i)}
$$

и

$$
\sum_{i=1}^{v} p_{i}=\sum_{i=1}^{v} p_{Z(i)}=1-p^{*}
$$

To

$$
\sum_{i=1}^{v} p_{i} p_{Z(i)}<\left(\sum_{i=1}^{v} p_{i}\right)^{2}=\left(1-p^{*}\right)^{2} \leqslant(1-\mathbf{P}(Z))^{2} .
$$

Таким образом, из неравенств

$$
\left(p^{*}\right)^{2} \leqslant \mathbf{P}(Z)<\left(p^{*}\right)^{2}+(1-\mathbf{P}(Z))^{2}
$$

следует, что

$$
p^{*}=(\mathbf{P}(Z))^{1 / 2}+O\left((1-\mathbf{P}(Z))^{2}\right)
$$

при $\mathbf{P}(Z) \rightarrow 1$ и

$$
(|\ln \mathbf{P}(Z)|)^{-1} \ln \left(\left(\mathbf{P}(Z)^{1 / 2}\left(p^{*}\right)^{-1}\right)=O(1-\mathbf{P}(Z)) \rightarrow 0,\right.
$$

T. e.

$$
\gamma(N)=O\left((1-\mathbf{P}(Z)) \frac{\ln N}{\ln \ln N}\right) .
$$

Из определения величины $n(x)$ следует, что

$$
n(x)=O\left(\frac{\ln C_{N}^{2}(1-\mathbf{P}(Z))}{1-\mathbf{P}(Z)}\right)=O\left(\frac{\ln N}{1-\mathbf{P}(Z)}+\frac{\ln (1-\mathbf{P}(Z))}{1-\mathbf{P}(Z)}\right)=O\left(\frac{\ln N}{1-\mathbf{P}(Z)}\right)
$$

и

$$
\frac{k}{n(x) \sqrt{1-p^{*}}}=O\left(\frac{k(1-\mathbf{P}(Z))}{\sqrt{1-p^{*}} \ln N}\right)=O\left(\frac{k\left(1-\left(p^{*}\right)^{2}\right)}{\sqrt{1-p^{*}} \ln N}\right)=O\left(\frac{k}{\ln N}\right) \rightarrow 0,
$$

т. е. выполняется условие $k / n\left(1-p^{*}\right)^{-1 / 2}<1$ из п. (b2) теоремы 1 . 
Справедливы тождества

$$
\begin{aligned}
-\frac{\ln p^{*}}{\ln \mathbf{P}(Z)} & =-\frac{1}{2}+\frac{1}{\ln \mathbf{P}(Z)} \ln \left(\frac{\sqrt{\mathbf{P}(Z)}}{p^{*}}\right), \\
-2 \ln N \frac{\ln p^{*}}{\ln \mathbf{P}(Z)} & =-\ln N+2 \gamma(N)(\ln \ln N) .
\end{aligned}
$$

Из (24) и условий теоремы вытекают оценки

$$
\begin{aligned}
\left(p^{*}\right)^{n(x)}= & \exp \left\{-\frac{\ln p^{*}}{\ln \mathbf{P}(Z)}\left(x+\ln \left(C_{N}^{2}(1-\mathbf{P}(Z))\right)\right\}\right. \\
= & \exp \left\{-\frac{1}{2}+\frac{1}{\ln \mathbf{P}(Z)} \ln \left(\frac{\sqrt{\mathbf{P}(Z)}}{p^{*}}\right)\left(x+\ln \left(C_{N}^{2}(1-\mathbf{P}(Z))\right)\right\}\right. \\
= & O\left(\left(C_{N}^{2}(1-\mathbf{P}(Z))\right)^{-1 / 2} \exp \left\{\frac{\ln \left(C_{N}^{2}(1-\mathbf{P}(Z))\right)}{\ln \mathbf{P}(Z)} \ln \frac{\sqrt{\mathbf{P}(Z)}}{p^{*}}\right\}\right) \\
= & O\left(\left(C_{N}^{2}(1-\mathbf{P}(Z))\right)^{-1 / 2} \exp \left\{\frac{\ln N}{\ln \mathbf{P}(Z)} \ln \frac{\sqrt{\mathbf{P}(Z)}}{p^{*}}\right\}\right. \\
& \left.\times \exp \left\{\left(\frac{1}{2}-\frac{\ln p^{*}}{\ln \mathbf{P}(Z)}\right) \ln (1-\mathbf{P}(Z))\right\}\right)
\end{aligned}
$$

и

$$
\begin{aligned}
\exp \left\{\frac{\ln N}{\ln \mathbf{P}(Z)} \ln \frac{\sqrt{\mathbf{P}(Z)}}{p^{*}}\right\} & =\exp \left\{\ln N\left(\frac{1}{2}-\frac{\ln p^{*}}{\ln \mathbf{P}(Z)}\right)\right\} \\
& =\exp \{\gamma(N)(\ln \ln N)\}=(\ln N)^{-\gamma(N)}, \\
\exp \left\{\left(\frac{1}{2}-\frac{\ln p^{*}}{\ln \mathbf{P}(Z)}\right) \ln (1-\mathbf{P}(Z))\right\} & =(1-\mathbf{P}(Z))^{1 / 2-c},
\end{aligned}
$$

где

$$
1 / 2<c=\frac{\ln p^{*}}{\ln \mathbf{P}(Z)} \leqslant 1
$$

Таким образом,

$$
\left(p^{*}\right)^{n(x)}=O\left(N^{-1}(1-\mathbf{P}(Z))^{-c}(\ln N)^{-\gamma(N)}\right)
$$

и

$$
n^{q}(x) N k\left(p^{*}\right)^{n(x)}=O\left(\frac{k}{(1-\mathbf{P}(Z))^{q+c}}(\ln N)^{q-\gamma(N)}\right) \rightarrow 0
$$

для любого $q>0($ так как $\gamma(N)=O((1-\mathbf{P}(Z)) \ln N /(\ln \ln N) \rightarrow \infty)$.

Таким образом, для случайной величины $\tilde{\xi}\left(L_{k v}, n(x), N\right)$ верна теорема 1 , т. е. $\mathbf{P}\left(\tilde{\xi}\left(L_{k v}, n(x), N\right)=0\right) \rightarrow e^{-\lambda}$.

Для любого положительного числа $a$ справедливо неравенство

$$
\mathbf{P}\left(\xi\left(L_{k v},[a]+1, N\right)=0\right) \geqslant \mathbf{P}\left(\mu\left(L_{k v}, N\right)<a\right) \geqslant \mathbf{P}\left(\xi\left(L_{k v},[a], N\right)=0\right) .
$$


Из (23) и теоремы 1 следует, что для целого $n \geqslant 1$

$$
\mathbf{P}\left(\xi\left(L_{k v}, n, N\right)=0\right)=\mathbf{P}\left(\tilde{\xi}\left(L_{k v}, n, N\right)=0\right) \rightarrow e^{-\lambda},
$$

где $C_{N}^{2} \psi\left(L_{k v}, n, \bar{p}\right) \rightarrow \lambda$.

Так как $\psi\left(L_{k v}, n, \bar{p}\right) \sim \psi\left(L_{k v}, n+1, \bar{p}\right)$ при $\mathbf{P}\left(Z_{m}\right) \rightarrow 1$, то

$$
\mathbf{P}\left(\tilde{\xi}\left(L_{k v}, n+1, N\right)=0\right) \sim \mathbf{P}\left(\tilde{\xi}\left(L_{k v}, n, N\right)=0\right)
$$

и

$$
\mathbf{P}\left(\mu\left(L_{k v}, N\right)<n\right) \sim \mathbf{P}\left(\tilde{\xi}\left(L_{k v}, n, N\right)=0\right) .
$$

В условиях теоремы это означает, что

$$
\mathbf{P}\left(\mu\left(L_{k v}, N\right)<n(x)\right) \sim \mathbf{P}\left(\tilde{\xi}\left(L_{k v}, n(x), N\right)=0\right) \rightarrow \exp \left\{-e^{-x}\right\} .
$$

Рассмотрим случай (b). Достаточно показать (см. [1]), что при дополнительном условии $\{2 \ln N /|\ln \mathbf{P}(Z)|\} \rightarrow \lambda_{0} \in[0,1]$

$$
\mathbf{P}\left(\mu\left(L_{k v}, N\right)-\left[\frac{2 \ln N}{|\ln \mathbf{P}(Z)|}\right]<d\right) \rightarrow \exp \left\{-\frac{1-\delta}{2} \delta^{d-\lambda_{0}}\right\}, \quad d=0, \pm 1, \ldots
$$

Действительно, так как

$$
\mathbf{P}\left(\mu\left(L_{k v}, N\right)-\left[\frac{2 \ln N}{|\ln \mathbf{P}(Z)|}\right]<d\right)=\mathbf{P}\left(\tilde{\xi}\left(L_{k v}, n(d), N\right)=0\right),
$$

где

$$
n(d)=[2 \ln N /|\ln \mathbf{P}(Z)|]+d
$$

то достаточно показать, что величины $n(d), k, v, p_{1}, \ldots, p_{v}$ и подстановки множества $L_{k v}$ удовлетворяют условиям теоремы 1 с параметром $\lambda=(1-\delta) \delta^{d-\lambda_{0}} / 2$.

Учитывая выражение для $n(d)$, получаем, что

$$
\begin{aligned}
C_{N}^{2} \psi\left(L_{k v}, n(d), \bar{p}\right) & =C_{N}^{2}(\mathbf{P}(Z))^{n(d)}(1-\mathbf{P}(Z)) \\
& =C_{N}^{2}(1-\mathbf{P}(Z)) \exp \left\{\left(-\frac{2 \ln N}{\ln \mathbf{P}(Z)}+2-\left\{-\frac{2 \ln N}{\ln \mathbf{P}(Z)}\right\}\right) \ln \mathbf{P}(Z)\right\} \\
& =\frac{1}{2} \delta^{d}(1-\delta)(\mathbf{P}(Z))^{-\{2 \ln N /|\ln \mathbf{P}(Z)|\}} \rightarrow \frac{1-\delta}{2} \delta^{d-\lambda_{0}}
\end{aligned}
$$

Так как $\mathbf{P}(Z)<p^{*}$ и $\mathbf{P}(Z) \rightarrow \delta \in(0,1)$, то $\lim \inf p^{*}>0$. Поэтому, учитывая это неравенство, тождества $(24)$ и условия теоремы, получаем, что

$$
\begin{aligned}
n^{q}(d)= & O\left(\left(-\frac{2 \ln N}{\ln \mathbf{P}(Z)}\right)^{q}\right)=O\left(\ln ^{q} N\right) \\
\left(p^{*}\right)^{n(d)}=\exp \left\{n(d) \ln p^{*}\right\}=\exp \left\{\left(d-\left\{-\frac{2 \ln N}{\ln \mathbf{P}(Z)}\right\}\right) \ln p^{*}\right\} & \\
& \quad \times \exp \left\{-\frac{2 \ln N}{\ln \mathbf{P}(Z)} \ln p^{*}\right\}=O\left(N^{-1}(\ln N)^{2 \gamma(N)}\right), \\
n^{q}(d) n k\left(p^{*}\right)^{n(d)}= & O\left(\left(\ln ^{q} N\right) k(\ln N)^{2 \gamma(N)}\right) \rightarrow 0
\end{aligned}
$$


для любого $q>0$.

Учитывая, что $k / \ln N \rightarrow 0$, аналогично проверяем условие

$$
k^{q} N\left(p^{*}\right)^{n(d)} \rightarrow 0
$$

Наконец, рассмотрим случай (c). Для целых значений числа $d$ с учетом (23) имеет место равенство

$$
\mathbf{P}\left(\mu\left(L_{k v}, N\right)=d\right)=\mathbf{P}\left(\tilde{\xi}\left(L_{k v}, d+1, N\right)=0\right)-\mathbf{P}\left(\tilde{\xi}\left(L_{k v}, d, N\right)=0\right) .
$$

Пусть $a=2 \ln N /|\ln \mathbf{P}(Z)|$, тогда из доказательства теоремы 1 (см. (16)) следует, что

$$
\mathbf{E} \tilde{\xi}\left(L_{k v},\langle a\rangle \pm 1, N\right)=C_{N}^{2} \psi\left(L_{k v},\langle a\rangle \pm 1, \bar{p}\right)+O\left(k(\langle a\rangle \pm 1) N\left(p^{*}\right)^{\langle a\rangle \pm 1}\right)
$$

С учетом этого соотношения, аналогично теореме 2 из [2], показывается, что

$$
\mathbf{P}\left(\xi\left(L_{k v},\langle a\rangle-1, N\right)=0\right) \rightarrow 0, \quad \mathbf{P}\left(\xi\left(L_{k v},\langle a\rangle+1, N\right)=0\right) \rightarrow 1 .
$$

Покажем, что для случайной величины $\xi\left(L_{k v},\langle a\rangle, N\right)$ справедлива теорема 1. Действительно,

$$
C_{N}^{2} \psi\left(L_{k v},\langle a\rangle, N\right) \sim C_{N}^{2}(\mathbf{P}(Z))^{\langle a\rangle} \sim C_{N}^{2} \exp \{a \ln \mathbf{P}(Z)\} \exp \{(a-\langle a\rangle)|\ln P(Z)|\} \rightarrow \lambda .
$$

Далее, с учетом (24) находим, что

$$
\left(p^{*}\right)^{a}=\exp \left\{-2 \ln N \frac{\ln p^{*}}{\ln \mathbf{P}(Z)}\right\}=O\left(N^{-1}(\ln N)^{2 \gamma(N)}\right) .
$$

Так как $a-\langle a\rangle \sim \ln (2 \lambda) /|\ln \mathbf{P}(Z)|$ и $\left|\ln p^{*}\right|<|\ln \mathbf{P}(Z)|$, то

$$
\left(p^{*}\right)^{\langle a\rangle-a}=\exp \left\{\ln (2 \lambda) \frac{\ln p^{*}}{\ln \mathbf{P}(Z)}\right\}=O(1)
$$

и

$$
\langle a\rangle^{q} k n\left(p^{*}\right)^{\langle a\rangle}=O\left(\left(\frac{\ln N}{|\ln \mathbf{P}(Z)|}\right)^{q} k(\ln N)^{2 \gamma(N)}\right) \rightarrow 0
$$

для любого $q>0$.

Аналогично показывается, что

$$
\langle a\rangle k^{q} N\left(p^{*}\right)^{\langle a\rangle}=O\left(\frac{\ln N}{|\ln \mathbf{P}(Z)|} k^{q}(\ln N)^{2 \gamma(N)}\right) \rightarrow 0
$$

для любого $q>0$.

Для второго случая условия (c) соотношения $\lambda([a]+1)=o(1)$ и $\lambda([a]) \rightarrow \infty$ проверяются аналогично. Далее,

$$
\begin{aligned}
{[a]^{q} k N\left(p^{*}\right)^{[a]} } & =o\left(\left(\frac{\ln N}{|\ln \mathbf{P}(Z)|}\right)^{q} k(\ln N)^{2 \gamma(N)}(\mathbf{P}(Z))^{-\{a\}}\right) \\
& =o\left(\left(\frac{\ln N}{|\ln \mathbf{P}(Z)|}\right)^{q} k^{q}(\ln N)^{2 \gamma(N)} \ln ^{b} N\right)=o(1)
\end{aligned}
$$

и

$$
[a] k^{q} N\left(p^{*}\right)^{[a]}=o\left(\frac{\ln N}{|\ln \mathbf{P}(Z)|} k^{q}(\ln N)^{2 \gamma(N)} \ln ^{b} N\right)=o(1)
$$




\section{6. Доказательство теоремы 4}

Для доказательства теоремы 4 достаточно показать, что (см. [5])

$$
\begin{gathered}
\exp \{2 \lambda(N)\} \max _{i, j} \mathbf{P}\left(\tilde{\eta}_{i j}(n)=1\right) \rightarrow 0, \\
\frac{1}{b !} \sum_{I_{m+b}(n, N)} \mathbf{P}\left(\alpha_{m+b}\right)=o\left(\lambda^{m-1}(N) e^{-\lambda(N)}\right), \\
\frac{1}{b !} \sum_{I_{m+b}(n, N)} \mathbf{P}\left(\tilde{\eta}_{i_{1} j_{1}}(n)=1\right) \ldots \mathbf{P}\left(\tilde{\eta}_{i_{m+b} j_{m+b}}(n)=1\right)=o\left(\lambda^{m-1}(N) e^{-\lambda(N)}\right)
\end{gathered}
$$

равномерно по $m=1, \ldots, 2[\lambda(N)]$ и по $b=0, \ldots,[9 \lambda(N)]-m$, и что

$$
\frac{\mathbf{P}\left(\tilde{\eta}_{i_{1} j_{1}}(n)=\ldots=\tilde{\eta}_{i_{r} j_{r}}(n)=1\right)}{\mathbf{P}\left(\tilde{\eta}_{i_{1} j_{1}}(n)=1\right) \ldots \mathbf{P}\left(\tilde{\eta}_{i_{r} j_{r}}(n)=1\right)}=1+o\left(e^{-2 \lambda(N)}\right)
$$

равномерно по $\alpha_{r} \notin I_{r}(n, N)$ и по $r=1, \ldots,[9 \lambda(N)]$.

В нашем случае при $\alpha_{r} \notin I_{r}(n, N)$ в силу независимости элементов последовательности $X$

$$
\mathbf{P}\left(\tilde{\eta}_{i_{1} j_{1}}(n)=\ldots=\tilde{\eta}_{i_{r} j_{r}}(n)=1\right)=\mathbf{P}\left(\tilde{\eta}_{i_{1} j_{1}}(n)=1\right) \ldots \mathbf{P}\left(\tilde{\eta}_{i_{r} j_{r}}(n)=1\right)
$$

и соотношение (28) выполнено.

Рассмотрим случай (a).

Из условий (7) и (8) вытекает, что

$$
\frac{k n\left(p^{*}\right)^{n}}{N \psi\left(L_{k v}, n, \bar{p}\right)}=O\left(\frac{n}{\lambda(N) N^{\delta}}\right) \rightarrow 0 .
$$

Поэтому с учетом (5), (6), (29) и доказательства теоремы 1 находим, что

$$
\begin{aligned}
\mathbf{E} \tilde{\xi}\left(L_{k v}, n, N\right) & =\sum_{1 \leqslant i<j \leqslant N-n+1} \mathbf{P}\left(\tilde{\eta}_{i j}(n)=1\right)=C_{N}^{2} \psi\left(L_{k v}, n, \bar{p}\right)+O\left(k n N\left(p^{*}\right)^{n}\right) \\
& =C_{N}^{2} \psi\left(L_{k v}, n, \bar{p}\right)\left(1+O\left(\frac{k n\left(p^{*}\right)^{n}}{N \psi\left(L_{k v}, n, \bar{p}\right)}\right)\right) \\
& =C_{N}^{2} \psi\left(L_{k v}, n, \bar{p}\right)(1+o(1))=\lambda(N)(1+o(1)) \rightarrow \infty .
\end{aligned}
$$

Поскольку из (6) следует, что

$$
\exp \{\lambda(N)\}=N^{\varepsilon /(\ln \lambda(N))},
$$

где $\varepsilon=\varepsilon(N) \rightarrow 0, \mathrm{a}$

$$
\max _{i, j} \mathbf{P}\left(\tilde{\eta}_{i j}(n)=1\right)<k\left(p^{*}\right)^{n}
$$

в силу (7) выполняется соотношение (25).

Нетрудно заметить, что с учетом возможных равенств $i_{s}=j_{t}$ или $j_{s}=j_{t}$ для некоторых пар индексов $\left(i_{s}, j_{s}\right),\left(i_{t}, j_{t}\right)$ из $\alpha_{r} \in I_{r}^{(a)}(n, N)$ справедливы оценки (здесь 
$(2 r-t)$ - число различных индексов набора $\left.\alpha_{r}\right)$

$$
\begin{aligned}
\left|I_{r}^{(a)}(n, N)\right| & <N^{a} \sum_{t=0}^{r-1} \sum_{\substack{s_{1}+\ldots+s_{a}=2 r-t \\
s_{1}, \ldots, s_{a}>0}} s_{1} ! \ldots s_{a} ! n^{2 r-a-t} \\
& <n^{2 r-a} N^{a} \sum_{t=0}^{r-1}\left(\begin{array}{c}
2 r-t-1 \\
a-1
\end{array}\right)(2 r-t) !<n^{2 r-a} N^{a}\left(\begin{array}{c}
2 r-1 \\
a-1
\end{array}\right) \sum_{t=0}^{r-1}(2 r-t) ! \\
& <n^{2 r-a} N^{a}\left(\begin{array}{c}
2 r-1 \\
a-1
\end{array}\right)(2 r) ! \sum_{t=0}^{r-1}(r+2)^{-t}<c_{1} n^{2 r-a} N^{a}\left(\begin{array}{c}
2 r-1 \\
a-1
\end{array}\right)(2 r) !
\end{aligned}
$$

(здесь и далее $c_{i}, i=1,2, \ldots$, - некоторые константы; напомним также, что $\left.I_{r}^{(a)}(n, N)=\left\{\alpha_{r} \in I_{r}: A\left(\alpha_{r}\right)=a\right\}\right)$.

Пусть

$$
q=\left(\frac{\psi}{1-p^{*}}\right)^{1 / 2} \frac{k N}{n}=O\left(\frac{\lambda(N)^{1 / 2}}{\left(1-p^{*}\right)^{1 / 2}} \frac{k}{n}\right),
$$

из (8) следует, что $q \rightarrow \infty$.

Условие (2) обеспечивает справедливость леммы 2 , поэтому для $\alpha_{r} \in I_{r}^{(a)}(n, N)$

$$
\mathbf{P}\left(\alpha_{r}\right)<\left(\frac{\psi}{1-p^{*}}\right)^{(a-1) / 2} k^{a}\left(p^{*}\right)^{n} .
$$

Далее, с учетом (30), неравенства $p^{*}<N^{-1-\delta}$ и соотношения $q \rightarrow \infty$ получаем, что

$$
\begin{aligned}
\sum_{I_{r}(n, N)} \mathbf{P}\left(\alpha_{r}\right) & =\sum_{a=1}^{2 r-1} \sum_{\alpha_{r} \in I_{r}^{(a)}(n, N)} \mathbf{P}\left(\alpha_{r}\right) \\
& <c_{1} \sum_{a=1}^{2 r-1} n^{2 r-a} N^{a}\left(\frac{\psi}{1-p^{*}}\right)^{(a-1) / 2}\left(p^{*}\right)^{n} k^{a}(2 r) !\left(\begin{array}{c}
2 r-1 \\
a-1
\end{array}\right) \\
& =c_{1} n^{2 r-1}(2 r) !\left(p^{*}\right)^{n} N k \sum_{a=0}^{2 r-2} q^{a}\left(\begin{array}{c}
2 r-1 \\
a
\end{array}\right) \\
& <c_{1} n^{2 r-1}(2 r) !\left(p^{*}\right)^{n} N k q^{2 r-1}\left(1+\frac{1}{q}\right)^{2 r-1} \\
& <c_{2} \lambda^{r-1 / 2}(N) N^{-\delta}\left(\frac{2 r^{2} k^{2}}{\left(1-p^{*}\right)}\left(1+\frac{1}{q}\right)^{2}\right)^{r}, \quad r=2, \ldots,[9 \lambda(N)] .
\end{aligned}
$$

Так как $\left(p^{*}\right)^{n} \rightarrow 0$, то $1 /\left(1-p^{*}\right)<c_{3} n$. Используя эти оценки, условие $(6)$, которое можно переписать в виде $N=\lambda(N)^{\lambda(N) / \varepsilon}(\varepsilon=\varepsilon(N) \rightarrow 0)$, и условия (8) и (9), легко проверить справедливость (26):

$$
\begin{aligned}
\frac{1}{b !} \sum_{I_{m+b}(n, N)} \mathbf{P}\left(\alpha_{m+b}\right) & <\lambda^{m-1}(N) e^{-\lambda(N)} c_{4}\left(c_{5}(\lambda(N))^{c_{6}-\delta / \varepsilon} k^{c_{7}} n^{c_{8}}\right)^{\lambda(N)} \\
& =o\left(\lambda^{m-1}(N) e^{-\lambda(N)}\right) .
\end{aligned}
$$


Пусть

$$
I_{r}(n, N)=\bigcup_{a=0}^{r} I_{r}(a ; n, N),
$$

где $I_{r}(a ; n, N)=\left\{\alpha_{r} \in I_{r}(n, N)\right.$ : имеется ровно $a$ таких значений $s, 1 \leqslant s \leqslant r$, что $\left.i_{s}<j_{s} \leqslant i_{s}+n\right\}$.

Нетрудно заметить, что

$$
\begin{aligned}
& \left|I_{r}(a ; n, N)\right|=O\left(n^{a} N^{2 r-a}(2 r) !\left(\begin{array}{l}
r \\
a
\end{array}\right)\right), \quad a=1, \ldots, r \\
& \left|I_{r}(0 ; n, N)\right|=O\left(n N^{2 r-a}(2 r) !\right) .
\end{aligned}
$$

Далее, из оценок (15) следует, что для $\alpha_{r} \in I_{r}(a ; n, N)$

$$
\mathbf{P}\left(\tilde{\eta}_{i_{1} j_{1}}(n)=1\right) \ldots \mathbf{P}\left(\tilde{\eta}_{i_{r} j_{r}}(n)=1\right) \leqslant\left(\left(p^{*}\right)^{n} k\right)^{a} \psi^{r-a} \text {. }
$$

Учитывая (29), получаем, что

$$
\begin{aligned}
\frac{1}{b !} \sum_{I_{r}(n, N)} \mathbf{P}\left(\tilde{\eta}_{i_{1} j_{1}}(n)=1\right) \ldots \mathbf{P}\left(\tilde{\eta}_{i_{r} j_{r}}(n)=1\right) \\
\quad \\
\quad<c_{9}\left(\lambda^{r}(N)\left(2 r^{2}\right)^{r} \frac{n}{N}+\lambda^{r}(N)\left(4 r^{2}\right)^{r} \frac{n k\left(p^{*}\right)^{n}}{N \psi}\right)<c_{10} \lambda^{r}(N)\left(4 r^{2}\right)^{r}\left(\begin{array}{c}
n \\
N^{\delta}
\end{array}\right)
\end{aligned}
$$

для всех $r=2, \ldots,[9 \lambda(N)]$. Из этих оценок с учетом соотношения $N=\lambda(N)^{\lambda(N) / \varepsilon}$ вытекает справедливость (27).

Рассмотрим случай (b).

Условие (1) обеспечивает справедливость леммы 1. Учитывая, что (здесь и далее $c_{i}, i=1,2, \ldots$, - некоторые константы)

$$
\psi_{1}<\frac{1}{1-p^{*}} \psi<c_{1} n \psi
$$

для $\alpha_{r} \in I_{r}^{(a)}(n, N), r \geqslant 2,1 \leqslant a \leqslant 2 r-1$, получаем оценку

$$
\mathbf{P}\left(\alpha_{r}\right)<\left(c_{2} n \psi\right)^{(a-1) / 2} k\left(p^{*}\right)^{n}
$$

Пусть $q_{1}=\left(c_{3} \lambda(N) / n\right)^{1 / 2}$, из (10) следует, что $q_{1} \rightarrow \infty$. Поэтому соотношение (26) принимает вид

$$
\begin{aligned}
\sum_{I_{r}(n, N)} \mathbf{P}\left(\alpha_{r}\right) & =\sum_{a=1}^{2 r-1} \sum_{\alpha_{r} \in I_{r}^{(a)}(n, N)} \mathbf{P}\left(\alpha_{r}\right)<n^{2 r-1}(2 r) !\left(p^{*}\right)^{n} N k \sum_{a=0}^{2 r-2} q_{1}^{a}\left(\begin{array}{c}
2 r-1 \\
a
\end{array}\right) \\
& <n^{2 r-1}(2 r) !\left(p^{*}\right)^{n} N k q_{1}^{2 r-1}\left(1+\frac{1}{q_{1}}\right)^{2 r-1} \\
& <c_{4} \lambda^{r-1 / 2}(N) N^{-\delta}\left(n r^{2}\left(1+\frac{1}{q_{1}}\right)^{2}\right)^{r}, \quad r=2, \ldots,[9 \lambda(N)] .
\end{aligned}
$$


Отсюда следует, что

$\frac{1}{b !} \sum_{I_{m+b}(n, N)} \mathbf{P}\left(\alpha_{m+b}\right)<\lambda^{m-1}(N) e^{-\lambda(N)} c_{5}\left(c_{6}(\lambda(N))^{c_{7}-\delta / \varepsilon} n^{c_{8}}\right)^{\lambda(N)}=o\left(\lambda^{m-1}(N) e^{-\lambda(N)}\right)$.

Условия (25), (27) и (28) проверяются аналогично.

Автор благодарен А.М. Зубкову за обсуждение результатов данной работы и ряд полезных замечаний.

\section{Список литературы}

1. Зубков А.М., Михайлов В.Г., Предельные распределения случайных величин, связанных с длинными повторениями в последовательности независимых испытаний. Теория вероятностей и ее применения (1974) 19, №1, 173-181.

2. Буравлев С.М., Повторения с точностью до перестановок в последовательности независимых испытаний. Дискретная математика (1999) 11, №1, 53-75.

3. Сачков В.Н., Комбинаторные методы дискретной математики. Наука, Москва, 1977.

4. Севастьянов Б.А., Предельный закон Пуассона в схеме сумм зависимых случайных величин. Теория вероятностей и ее применения (1972) 17, №4, 333-338.

5. Амбросимов А.С., Нормальный закон в схеме сумм зависимых случайных величин, рассмотренных Б.А. Севастьяновым. Теория вероятностей и ее применения (1976) 21, №1, 184-189.

Статья поступила 24.06.1999. 